\title{
Problèmes de reconstitution d'éprouvettes de sols argileux en laboratoire.
}

\author{
Assia BAT \\ Université du HAVRE et CETE de ROUEN
}

\section{Résumé}

Pour faire varier les procédures d'essai et voir leurs influences sur les paramètres mesurés en laboratoire, il convient d'avoir des éprouvettes reproductibles ou, au moins, ayant un faible taux de dispersion. Pour ce faire il faut, soit prélever un sol naturel peu hétérogène, soit reconstituer des éprouvettes. Le choix du mode de confection des éprouvettes est à faire entre la confection à l'unité et la réalisation de massifs dans de gros oedomètres, dans lesquels les éprourvettes seront carottés. Pour pallier les inconvénients qui pourraient résulter du carottage des éprouvettes et de leur taille, nous avons opté pour la confection à l'unité des échantillons par consolidation d'une pâte argileuse (kaolin), dans des moules aux dimensions des éprouvettes d'essais. Des appareils de consolidation, appelés consolidomètres, sont présentés ainsi que les résultats et les premières analyses relatives aux essais de consolidation.

\section{Obiectif:}

La validité des paramètres d'état des sols, mesurés au laboratoire, est conditionnée par le mode de prélèvement des échantillons ainsi que par les conditions imposées lors des procédures d'essais.

Lors d'une étude géotechnique, le prélèvement d'échantillon de sol est la première étape à soigner afin d'obtenir des résultats significatifs à l'issue d'essais bien adaptés et correctement menés. Le remaniement est à observer sur l'ensemble des opérations depuis le moment où l'échantillon est extrait de son gisement naturel, jusqu'à ce qu'il soit soumis aux essais en vue desquels il aura été prélevé. Ces opèrations sont : la prise d'échantillon, le conditionnement, le transport, le stockage, ainsi que la préparation de l'éprouvette et son montage dans l'appareil d'essai.

La fiabilité des paramètres d'états des sols naturels mesurés en laboratoire, est aussi liée à la précision des procédures d'essais (essais de cisaillement et essais de compressibilité). Les normes récemment éditées (NF P 94-070 et NF P 94-074) en octobre 1994 présentent des imprécisions quant à un certain nombre de conditions d'essais, telles que les valeurs et les variations de la température d'essai, l'utilisation du papier filtre et de la membrane isolant l'éprouvette pour les essais triaxiaux, le système de drainage, le système d'antifrettage, la mesure de la pression interstitielle, etc. 
Dans lobjectif d'essayer d'évaluer lincidence de ces procédures d'essais (essais triaxial et essai oedométrique) sur les résultats expérimentaux et sur les caractéristiques géotechniques mesurés en laboratoire, il semble à priori préférable de travailler sur des échantillons de sols reconstitués en laboratoire en se souciant principalement de la répétabilité et de l'homogénéité des éprouvettes obtenues.

En effet, l'hétérogénéité des sols naturels et linévitable remaniement dû aux différentes étapes du prélèvement d'échantillons de sol, sont responsables de dispersions souvent remarquées lors d'essais de laboratoire. Cette dispersion inhérente aux sols naturels nécessiterait d'effectuer un grand nombre d'essais pour obtenit des valeurs moyennes sur lesquels des comparaisons puissent avoir un sens.

\section{Travaux préparatoires: Réalisation d'un sol de référence.}

De nombreux laboratoires ont utilisé différentes méthodes de consolidation d'échantillons de sols naturels ou d'échantillons reconstitués. On dénombre ainsi différents modes de confection des éprouvettes qui se résument soit dans la réalisation de massifs de sol dans de gros oedomètres dans lesquels les éprouvettes seront carottées et taillées, soit dans la confection des échantillons par consolidation de pâte dans des moules de faibles dimensions.

\subsection{Eprouvettes au laboratoire}

Le Laboratoire Régional des Ponts et Chaussées d'Aix en Provence est liun des laboratoire ayant conçu un matériel de reconstitution d'échantillons de sol au laboratoire appelé consolidomètre. La boue utilisée est préparée à partir de sol naturel trituré, séché, tamisé à des dimensions de grains bien précises, puis malaxé ayec de l'eau à une teneur en eau de l'ordre de deux fois la limite de liquidité du sol. On dénombre deux consolidomètres à deux dimensions différentes.

*Le consolidomètre $(\varnothing 140)$ est constitué d'un piston et de deux corps cylindriques en PVC concentriques séparés par du géotextile. Le cylindre intérieur est percé aléatoirement de trous qui assurent un drainage latéral et fendu selon une génératrice pour faciliter la récupération de l'échantillon après la consolidation. La carotte récupérée est alors coupée et taillée en 4 éprouvettes, de diamètre $50 \mathrm{~mm}$ et d'élancement 2 pour les essais triaxiaux, sur lesquelles on constate une faible variation de l'indice des vides et une répétabilité assez satisfaisante.

* Le consolidomètre $(\varnothing 50)$ est constitué de deux pistons haut et bas et d'un corps cylindrique percé et habillé intérieurement de géotextile. Ce corps cylindrique rempli de boue est alors monté dans une cellule triaxiale à deux compartiments. Une fois l'éprouvette de diamètre $50 \mathrm{~mm}$ récupérée, il suffit simplement de couper le haut et le bas de l'éprouvette pour avoir l'élancement voulu. L'incommodité de ce dispositif, notamment l'utilisation d'une cellule triaxiale, a limité le nombre d'essais réalisés, et de ce fait les informations sur la répétabilité des échantillons consolidés. Dans les deux cas, tout le dispositif est placé sur un bâti oedométrique à poids, et la fin de l'essai est déterminée par une hauteur finale en fonction de la densité visée, ou par une charge finale pour une cohésion souhaitée. 


\subsection{Massifs d'essais}

La consolidation est également utilisée pour la reconstruction de massifs d'essais (cuve, modèles réduits centrifugés). Ainsi pour réaliser des massifs de kaolin pour modèles centrifugés, le L.C.P.C. à Nantes a procédé à la consolidation couche par couche, de hauteurs initiales de $170 \mathrm{~mm}$, dans un oedomètres de $900 \mathrm{~mm}$ de diamètre avec du kaolin de type Spes White (China clay de Cambridge).

La boue de kaolin se fait par mélanges intermédiaires de petites quantités d'eau et de kaolin malaxés sous vide à une teneur en eau de l'ordre de $98 \%$ (à peu près 2 fois la limite de liquidité du kaolin). Les couches du massif sont ensuite soumises à des paliers de charge, maintenus pendant 24 heures, au bout desquels on dépasse largement les $90 \%$ de consolidation. La cohésion est déterminée à l'aide d'un pénétromètre au lieu d'un scissomètre. Il existe une corrélation entre la cohésion $c_{u}$ et la résistance en pointe $q_{c}$ vérifiée expérimentalement et le profil de la cohésion en fonction de la profondeur est donné par : $c_{\mathrm{u}}(\mathrm{kPa})=1,75 \mathrm{z}(\mathrm{m})+5($ avec $\mathrm{z}$ la profondeur). Malgré la taille importante des massifs ainsi préparés, leur homogénéité reste très bonne et leur répétabilité assurée.

\section{Expérimentation au laboratoire}

\subsection{Idée de base}

II s'agit de disposer d'une méthode de confection d'éprouvettes d'argile par consolidation unidimensionnelle ou radiale. L'expérimentation au laboratoire consiste à tester de nombreuses éprouvettes, il importe donc que le dispositif et la méthode de préparation envisagée permettent d'assurer la répétabilité des éprouvettes, de produire des échantillons les plus homogènes possibles, et d'avoir un mode opératoire simple, insensible au facteur opérateur.

Le but est de reconstituer des éprouvettes aux dimensions des essais triaxiaux, de $50 \mathrm{~mm}$ de diamètre et d'élancement 2 , par consolidation de boue de teneur en eau comprise entre 1,5 et 2 fois la limite de liquidité du sol. La consolidation consistera à la sollicitation d'un échantillon cylindrique de kaolin saturé, en compression axiale et sans déformation latérale. L'écoulement de l'eau interstitielle sera de préférence à la fois vertical et latéral pour limiter la durée de la consolidation.

En ce qui concerne le matériau à utiliser, il est apparu souhaitable d'exploiter l'expérience acquise au L.C.P.C. à Nantes avec le kaolin de limite de liquidité $\mathrm{w}_{L}=49 \%$, préalablement malaxé sous vide, et stocké dans une chambre de saturation. Il faudra une contrainte de fin de consolidation de l'ordre de $220 \mathrm{kPa}$ pour une cohésion finale de $40 \mathrm{kPa}$.

\subsection{La réalisation de l'essai}

\subsubsection{Appareillage du consolidomètre de laboratoire}

Le consolidomètre est constitué d'un tube cylindrique, d'un piston supportant un plateau sur lequel seront placées les charges et d'une embase drainante rehaussée de papier filtre ou de géotextile, afin d'assurer le drainage vertical par le bas. 
Le piston est maintenu en position verticale dans un bâti en U par un système de guidage de hauteur de l'ordre de 3 fois le diamètre du piston. Pour mesurer le tassement en fin de consolidation (c'est-à-dire après dissipation de la surpression interstitielle), on dispose d'un réglet pour les premiers paliers et d'un comparateur pour les faibles tassements (Voir Fig. 1).
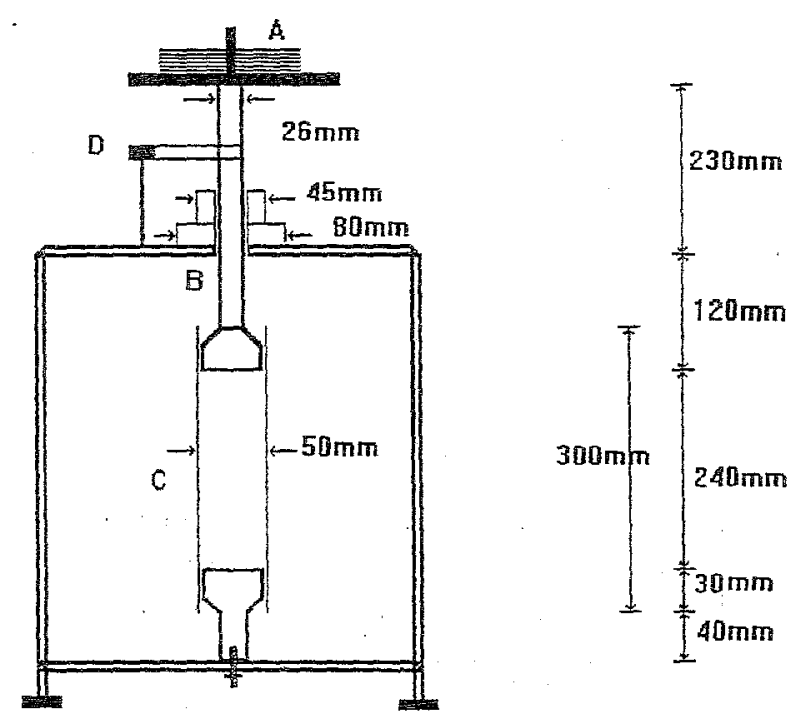
A : Plateau des poids
B : Piston
$\mathrm{C}$ : Consolidomètre en filtroplast, en PVC ou en acier.
D : Capteur de déplacement axial

Fig. 1 Dispositif de consolidation

Le chargement consiste à appliquer une série de contraintes verticales pendant 24 heures avec un taux d'accroissement de $0 ; 5$, constituant ainsi des paliers de charge. La première charge, qui correspond au poids propre du piston et du plateau, permet de corriger les défauts de surface de l'échantillon et la mise en place du piston sur l'éprouvette.

\subsubsection{Corps du consolidomètre}

*Le consolidomètre à niltroplast : Il est constitué par un cylindre en plastique fritté "Filitroplast". Ce plastique poreux est destiné à un large spectre d'applications (fluidisation, aération et filtration). L'eau pourra circuler aussi bien verticalement, que horizontalement vers les extrémités drainantes, tant qu'il n'y a pas colmatage des pores lors de la consolidation. Les caractéristiques géométriques du tube sont: diamètre intérieur $50 \mathrm{~mm}$, diamètre extérieur $60 \mathrm{~mm}$, diamètre des pores $35 \mu \mathrm{m}$ et la hauteur $300 \mathrm{~mm}$. 
* Le consolidomètre en PVC : identique au consolidomètre $\varnothing 50$ du L.R.P.C d'Aix en Provence habillé intérieurement de géotextile (Bidim) d'épaisseur $1 \mathrm{~mm}$ environ. Les caractéristiques géométriques du tube sont : diamètre intérieur $53,6 \mathrm{~mm}$, diamètre extérieur $60 \mathrm{~mm}$ et la hauteur $300 \mathrm{~mm}$.

* Le consolidomètre en acier : constitué d'un tube en acier inoxydable entouré de membrane en latex d'épaisseur de l'ordre de $0,3 \mathrm{~mm}$. La surface interne du tube en acier est préalablement enduite de lubrifiant afin de faciliter la récupération de l'échantillon après consolidation. Du vide est réalisé entre la membrane et la paroi interne du tube, tout en remplissant le consolidomètre de boue. Les dimensions géométriques du tube sont : diamètre intérieur $50,5 \mathrm{~mm}$, diamètre extérieur $65 \mathrm{~mm}$ et la hauteur $300 \mathrm{~mm}$. Le drainage sera seulement vertical, il faudra alors comparer les différents temps de consolidation nécessaires pour ces différents consolidomètres, afin de déterminer l'influence du chemin de drainage sur la qualité de l'éprouvette et sur la durée de la consolidation.

Tous ces consolidomètres seront montés sur des bâtis identiques, en prenant soin d'utiliser les pistons bas et haut correspondants.

\subsection{Présentation et analyse des résultats d'essais.}

Le première éprouvette consolidé dans un consolidomètre à filtroplast (filtroplast 1 ), sous une charge finale de $220 \mathrm{kPa}$, a un aspect relativement mou qui indique que la cohésion visée $(40 \mathrm{kPa})$ n'est pas atteinte. On pense alors à deux hypothèses : le dernier palier de chargement n'est probablement pas la sollicitation qu'il faut pour la cohésion recherché ou bien le colmatage des pores, durant la consolidation, empêche le drainage latéral.

Pour étayer ces hypothèses les essais filtroplast 2 et filtroplast 3 ont été effectués, en passant de $220 \mathrm{kPa}$ à $330 \mathrm{kPa}$. L'augmentation de la charge du dernier palier a changé l'état finale de l'éprouvette : diminution de l'indice des vides final et augmentation des densités humides et sèches finales sans pour autant atteindre la cohésion voulue (Voir Tableau. 1).

Remarques : II n'y a pas eu de tassement sous certains paliers, cela peut s'expliquer par un frottement, du piston sur les parois du consolidomètre, supérieur à la sollicitation du palier.

Tableau. 1 Récapitulatif des caractéristiques géotechniques des éprouvettes Consolidomètre à filtroplast

\begin{tabular}{|c|c|c|c|c|c|c|c|c|c|c|c|c|c|}
\hline & $\phi_{(\mathrm{mm})}$ & $\begin{array}{l}\mathrm{H}_{\mathrm{i}} \\
\text { (mm })\end{array}$ & $\underset{(\mathrm{mm})}{\mathrm{H}_{\mathrm{f}}}$ & $\begin{array}{l}w_{i} \\
(\%)\end{array}$ & $\begin{array}{l}\text { Wf } \\
(\%)\end{array}$ & $\begin{array}{l}\sigma_{\mathrm{V}}^{\prime} \\
(\mathrm{kPa})\end{array}$ & $\begin{array}{l}\mathrm{SI}_{1} \\
(\%)\end{array}$ & $\begin{array}{l}\mathrm{Sr}_{f} \\
(\%)\end{array}$ & $\mathrm{e}_{\mathrm{i}}$ & $e_{f}$ & $\begin{array}{c}\text { ef } \\
\text { calculé }\end{array}$ & $\begin{array}{c}\rho \mathrm{df} \\
\left(\mathrm{g}^{\prime} \mathrm{cm}^{3}\right)\end{array}$ & $\underset{(\mathrm{kPa})}{\mathrm{c}_{\mathrm{Pa}}}$ \\
\hline $\begin{array}{c}\text { Filtro } \\
\text { plast } \\
1\end{array}$ & 50 & 236 & 147 & 99,7 & 48,7 & 220 & 95,9 & 96,4 & 2,75 & 1,34 & 1,29 & 1,13 & - \\
\hline $\begin{array}{c}\text { Filtro } \\
\text { plast } \\
2\end{array}$ & 50 & 242 & 141 & 101,4 & 46,9 & 330 & 92,9 & 97,8 & 2,89 & 1,27 & 1,24 & 1,17 & - \\
\hline \begin{tabular}{|c|}
$\begin{array}{c}\text { Filtro } \\
\text { plast } \\
3\end{array}$ \\
\end{tabular} & 50 & 245 & 148 & 91,2 & 45,4 & 330 & 89,3 & 97,2 & 2,71 & 1,24 & 1,20 & 1,18 & $\begin{array}{l}5,8 \\
2,9 \\
1,3 \\
\end{array}$ \\
\hline
\end{tabular}


En fin de consolidation, l'éprouvette adhère fortement à la paroi interne du consolidomètre, ceci nécessite l'emploi d'un vérin pour l'extraire. L'échantillon subit alors un cisaillement latéral et un léger compactage. On mesure la cohésion, sur 3 niveaux de l'éprouvette (filtroplast 3 ), avec un scissomètre de laboratoire: les pales utilisées sont de diamètre $12,5 \mathrm{~mm}$ et d'élancement 2. Les valeurs de cohésion restent néanmoins 10 fois plus faibles que la cohésion prévue par le L.C.P.C à Nantes (Voir Tableau.1). On lance alors une série d'essais avec le consolidomètre en PVC (PVC1, PVC2 et PVC3). L'échantillon PVC1 de même teneur en eau initiale que le filtroplast 3 est dans un état de cohésion final légèrement inférieur à celui du filtroplast3. L'échantillon PVC2 est consolidé dans le consolidomètre en PVC, aléatoirement percé de petits trous de drainage sur 4 génératrices. On constate un changement du profil de cohésion dans l'éprouvette, mais un état de cohésion faible (Voir Tableau.2).

Tableau. 2 Récapitulatif des caractéristiques géotechniques des éprouvettes Consolidomètre en PVC

\begin{tabular}{|c|c|c|c|c|c|c|c|c|c|c|c|c|c|}
\hline & $\begin{array}{c}\phi \\
(\mathrm{mm})\end{array}$ & $\mathrm{H}_{1}$ & $\underset{(\mathrm{mm})}{\mathrm{Hf}}$ & $\begin{array}{l}W_{1}^{*} \\
(\%)\end{array}$ & $\begin{array}{l}w_{f} \\
(\%)\end{array}$ & $\begin{array}{r}\sigma_{V}^{\prime} \\
(\mathrm{kPa})\end{array}$ & $\begin{array}{l}\mathrm{Sr}_{1}^{\prime} \\
(\%)\end{array}$ & $\begin{array}{l}\mathrm{Srf} \\
(\%)\end{array}$ & $e_{j}$ & $e_{f}$ & $\underset{\text { calculé }}{e_{f}}$ & $\begin{array}{r}P \mathrm{df} \\
\left(\mathrm{g} / \mathrm{cm}^{3}\right)\end{array}$ & $\underset{(\mathrm{kPa})}{\mathrm{c}}$ \\
\hline $\begin{array}{c}\text { PVC } \\
1\end{array}$ & 51,5 & 235 & 136 & 91,2 & 47,4 & 311 & 75,5 & 87,9 & 3,20 & 1,43 & 1,26 & 1,09 & $\begin{array}{l}4,5 \\
2,5 \\
1,1\end{array}$ \\
\hline $\begin{array}{c}\text { PVC } \\
2\end{array}$ & 52 & 229 & 132 & 91,1 & 48 & 383 & 78,8 & 94,7 & 3,06 & 1,34 & 1,27 & 1,13 & $\begin{array}{l}3,8 \\
6,1 \\
3,8\end{array}$ \\
\hline $\begin{array}{c}\text { PVC } \\
3 \\
\end{array}$ & 51,5 & 244 & 139 & 105 & 55,9 & 463 & 70,9 & 79,2 & 3,92 & 1,80 & 1,48 & 0,97 & $\begin{array}{l}8,1 \\
4,9\end{array}$ \\
\hline
\end{tabular}

A ce stade des essais de consolidation, et du fait des résultats non concluants, on s'oriente vers une diminution des frottements du piston sur la paroi interne. $\mathrm{Ce}$ frottement peut conduire à une contrainte réellement appliquée sur le sol inférieure à la contrainte estimée. La charge serait alors supportée à la fois par le consolidomètre et par l'éprouvette. Une autre série d'essais est alors réalisée dans un oedomètre classiquie (Oedomètre 1 , Oedomètre 2 et Oedomètre 3 ) de hauteurs initiales $32,1 \mathrm{~mm}$ et de diamètres $70 \mathrm{~mm}$ (Voir Tableau.3). En fin de consolidation, l'état final de l'éprouvette oedomètrel, relativement molle, de hauteur finale de $20,2 \mathrm{~mm}$, n'est pas totalement satisfaisant.

Tableau. 3 Récapitulatif des caractéristiques géotechniques des éprouvettes Qedomètre classique

\begin{tabular}{|c|c|c|c|c|c|c|c|c|c|c|c|c|c|}
\hline & $\begin{array}{c}\phi \\
(\mathrm{mm})\end{array}$ & $\begin{array}{c}\mathrm{H}_{\mathrm{i}} \\
(\mathrm{mm})\end{array}$ & $\begin{array}{c}\mathrm{H}_{\mathrm{f}} \\
(\mathrm{mm})\end{array}$ & $\begin{array}{c}\mathrm{W}_{\mathrm{i}} \\
(\%)\end{array}$ & $\begin{array}{c}\mathrm{W}_{\mathrm{f}} \\
(\%)\end{array}$ & $\begin{array}{c}\sigma_{\mathrm{V}}^{\mathrm{V}} \\
(\mathrm{kPa})\end{array}$ & $\begin{array}{c}\mathrm{Sr}_{\mathrm{i}} \\
(\%)\end{array}$ & $\begin{array}{c}\mathrm{Sr}_{\mathrm{f}} \\
(\%)\end{array}$ & $\mathrm{e}_{\mathrm{i}}$ & $\mathrm{e}_{\mathrm{f}}$ & $\begin{array}{c}\mathrm{e}_{\mathrm{f}} \\
\mathrm{calcusic}\end{array}$ & $\begin{array}{c}\rho_{\mathrm{d} f} \\
\left(\mathrm{~g} / \mathrm{cm}^{3}\right)\end{array}$ & $\begin{array}{c}\mathrm{c}_{\mathrm{u}} \\
(\mathrm{kPa})\end{array}$ \\
\hline $\begin{array}{c}\text { Oedo } \\
\text { mètre1 }\end{array}$ & 70 & 32,1 & 20,2 & 91,1 & 44,7 & 307 & 97,1 & 99,3 & 2,49 & 1,20 & 1,19 & 1,21 & 13,7 \\
\hline $\begin{array}{c}\text { Oedo } \\
\text { mètre2 }\end{array}$ & 70 & 32,1 & 19,5 & 92,8 & 45,2 & 464 & 95,3 & 100 & 2,62 & 1,20 & 1,20 & 1,21 & 7,3 \\
\hline $\begin{array}{c}\text { Oedo } \\
\text { mètre3 }\end{array}$ & 70 & 32,1 & 20,2 & 91,9 & 46,8 & 464 & 95,6 & 99,6 & 2,57 & 1,25 & 1,24 & 1,18 & 21,5 \\
\hline
\end{tabular}


La mesure de la cohésion de l'oedomètrel se fait alors à l'aide de pales de diamètre $12,5 \mathrm{~mm}$ et d'élancement 1 (Voir Tableau.3). Les valeurs de cohésion ainsi mesurées constituent un ordre de grandeur, car cet essai est effectué dans des conditions hors norme.

La dernière série d'essais est réalisée dans un consolidomètre en Inox (Inoxl et Inox2). On constate, en fin d'essai, une relative fermeté de l'état de l'échantillon Inox 1: de cohésion finale allant de $37 \mathrm{kPa}$ en haut de l'éprouvette à $56 \mathrm{kPa}$ au milieu de l'éprouvette, et de $41 \mathrm{kPa}$ en bas de l'éprouvette (Voir Tableau 4.). Néanmoins après extraction des échantillons Inox, on remarque des vides sur la paroi latérales qui correspondent aux emplacements des plis de la membrane Latex. Ces vides engendrent forcement des erreurs dans le calcul des indices des vides finaux.

Tableau. 4 Récapitulatif des caractéristiques géotechniques des éprouvettes Consolidomètre en INOX

\begin{tabular}{|c|c|c|c|c|c|c|c|c|c|c|c|c|c|}
\hline & $\underset{(\mathrm{mm})}{\phi}$ & $\begin{array}{l}\mathrm{H}_{\mathrm{i}} \\
(\mathrm{mm})\end{array}$ & $\begin{array}{r}\mathrm{H}_{\mathrm{f}} \\
(\mathrm{mm})\end{array}$ & $\begin{array}{l}\mathrm{w}_{\mathrm{i}} \\
(\%)\end{array}$ & $\begin{array}{l}\text { Wf } \\
(\%)\end{array}$ & $\begin{array}{l}\sigma_{\mathrm{V}}^{\prime} \\
(\mathrm{kPa})\end{array}$ & $\begin{array}{l}\mathrm{Sr}_{\mathrm{i}} \\
(\%\end{array}$ & $\begin{array}{l}\operatorname{Srf}_{(\%)} \\
\text { (\%) }\end{array}$ & $e_{i}$ & $e_{f}$ & $\underset{\text { caicule }}{\mathrm{e}}$ & $\underset{\left(\mathrm{g} / \mathrm{cm}^{3}\right)}{\rho \mathrm{df}}$ & $\begin{array}{c}c_{4} \\
(\mathrm{kPa})\end{array}$ \\
\hline $\begin{array}{c}\text { INOX } \\
1\end{array}$ & $\longdiv { 4 9 , 9 }$ & 257 & 158 & 90,8 & 42,9 & 406 & 94,4 & 96,2 & 2,55 & 1,18 & 1,14 & 1,22 & $\begin{array}{l}37 \\
56 \\
41\end{array}$ \\
\hline $\begin{array}{c}\text { INOX } \\
2\end{array}$ & $\longdiv { 4 9 , 9 }$ & 252 & 163 & 90,7 & 45,3 & 406 & 95,5 & $\overline{99,6}$ & 2,52 & 1,28 & 1,20 & 1,20 & $\begin{array}{l}52 \\
44 \\
53\end{array}$ \\
\hline
\end{tabular}

Les échantillons PVC3, Oedomètre 3 et Inox2, à l'exception des autres éprouvettes, ont été réalisés avec du kaolin malaxé au laboratoire à partir d'une poudre kaolin de type Spes. White (China Clay de Cambridge). On constate alors que ces différents échantillons donnent le même état de cohésion que les échantillons issus des mêmes consolidomètres, avec une visible augmentation de lindice des vides final et de la teneur en eau finale (Voir les tableaux 2, 3 et 4). On suit la consolidation sous les derniers paliers de chargement pour s'assurer de la dissipation totale des surpressions interstitielles, et on trace les courbes du tassement $\Delta H$ en fonction du temps en mesurant le $\mathrm{t}_{100}$ de chaque courbe de consolidation. On constate alors que le $t_{100}$ le plus élevé correspond au consolidomètre en Inox, c'est à dire le chemin de drainage le plus long (Voir fig.2). Durant les essais de consolidation, on trace aussi les courbes de l'indice des vides e en fonction de la contrainte appliquée $\sigma^{\gamma}\left(\lg \sigma^{\prime}\right)$, et on détermine les pentes $C_{c}$ (indice de compression) de ces courbes de compressibilité (Voir fig. 3). Nous constatons que l'objectif visé, c'est à dire une répétabilité des échantillons, est loin d'être satisfait, comme le montre la dispersion constatée dans les résultats des différents consolidomètres. Les causes sont probablement les frottements des pistons sur les parois des consolidomètres et à l'accumulation d'incertitudes assez grandes, dues aux erreurs induites par la difficulté de remplissage des moules d'une part (mesures de $\mathrm{H}_{\mathrm{i}}$, $\mathrm{e}_{\mathrm{i}}$ et $\rho_{\mathrm{j}}$ ) et par la difficulté de mesurer avec précision les hauteurs finales d'un échantillon relativement mou d'autre part. De plus, ces difficultés propre à chaque outil de consolidation, ne permettent pas une 
comparaison constructive entre les différents échantillons issus des divers consolidomètres:

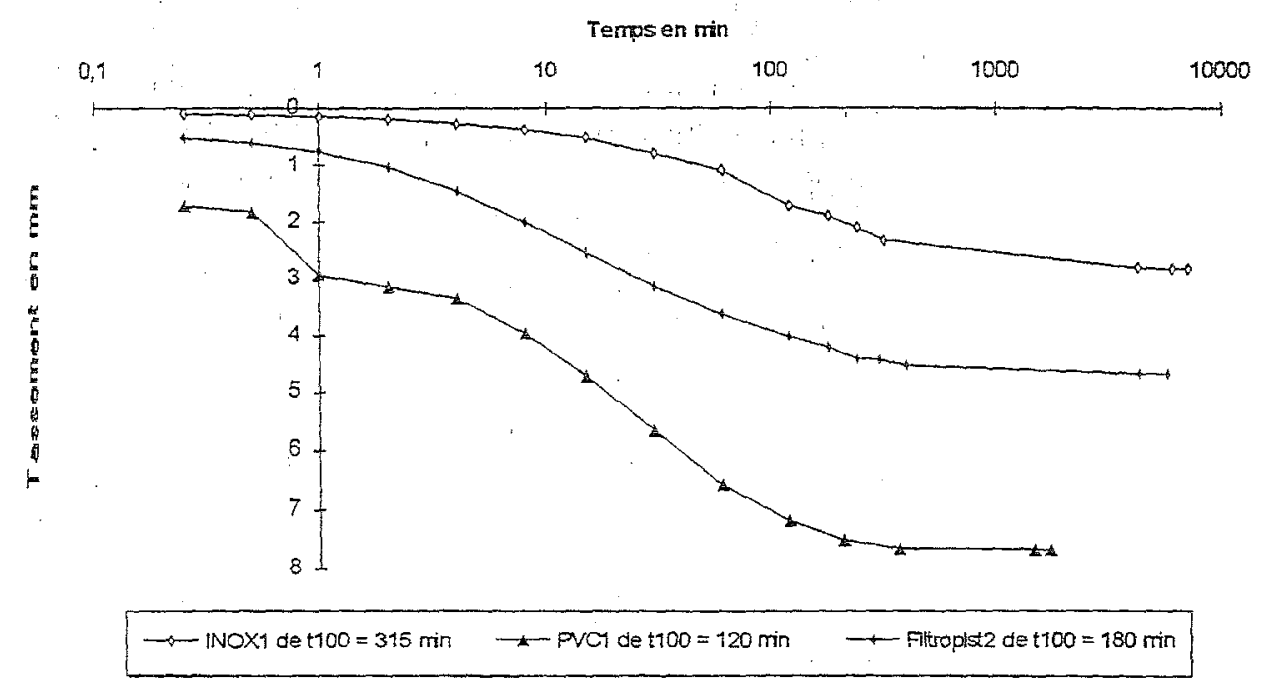

Fig. 2 Courbes de consolidation

Tassement en fonction du Temps

\section{Conclusion}

La reconstitution des échantillons au laboratoire ne semble pas répondre aux attentes concernant la qualité et la répétabilité des échantillons. En effet on constate une dispersion des valeurs des différentes caractéristiques géotechniques finales. On devra se toumer soit vers la consolidation dans des oedomètres de 300 $\mathrm{mm}$ de diamètre dans lesquels on prélève des éprouvettes en prenant le soin de les carotter loin des parois, soit vers le prélèvement d'échantillons "intacts" dans un site de sol fin le plus homogène possible, en multipliant le nombre d'essais de laboratoire.

Le choix d'éprouvettés de sol de référence, est une étape préliminaire très importante pour le bon déroulement d'un programme d'essais au laboratoire. Ainsi avant d'entamer les séries d'expériences sur linfluence des procédures d'essais sur les paramètres géotechniques il faut faire le meilleur choix entre les différents modes de confection des éprouvettes de sol au laboratoire (consolidomètres et massifs d'essai), et les prélèvements des échantillons "intacts". Le choix se fera principalement en fonction de lhomogénéité et de la répétabilité des éprouvettes obtenues.

\section{Références}

Norme française NF P 94_070 "Sols : Reconnaissance et essais. Essais à lappareil triaxial de révolution. Généralités-Définitions.". Octobre 1994, 21 pp.

Norme française NF P 94_074 "Sols : Reconnaissance et essais. Essais à l'appareil triaxial de révolution.Appareillage-Préparation des éprouvettes- Essai (UU)-Essai (CU+u) - Essai (CD).",Octobre 1994, 36 pp. 
Fig.3 Courbes de compressibilité

Indice des vides en fonction de la contrainte appliquée $\sigma^{\prime} \underline{y}$

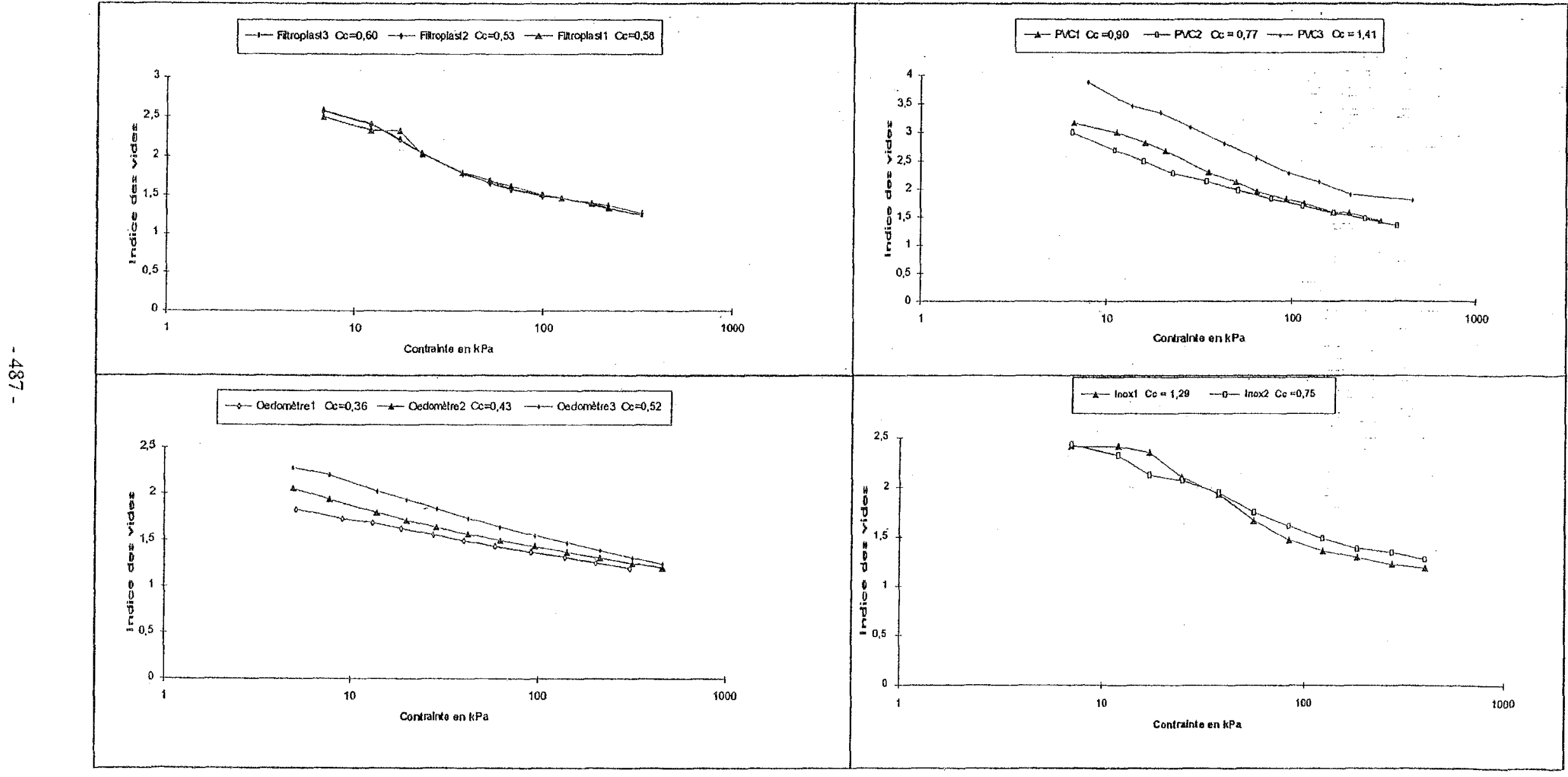

